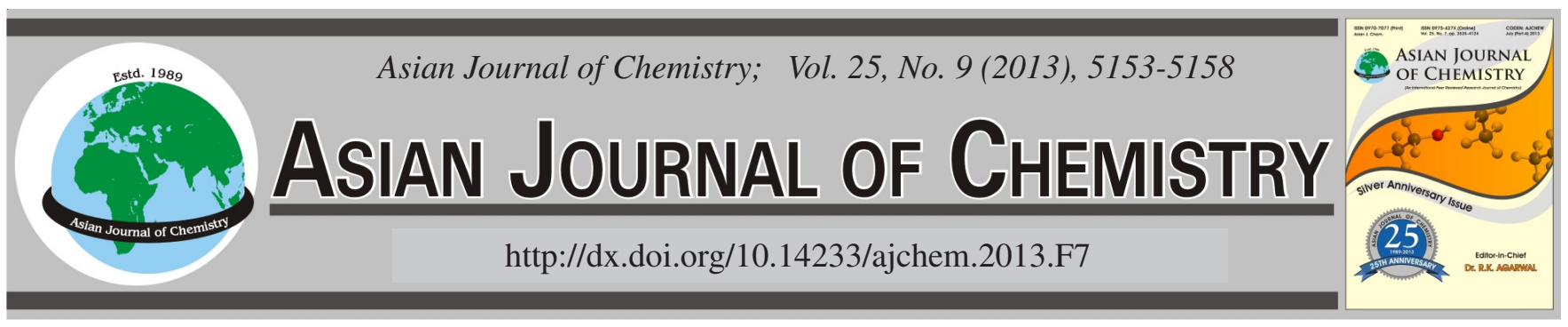

\title{
Investigation of the Mechanical, Electrical and Thermal Properties of Rubber Composites Filled with Various Carbon Nanomaterials, Carbon Nanotube, Graphene and Graphite $\dagger$
}

\author{
Sung-Woo Kim*, Ho-Kyun Jeong, Yong-Gu Kang and Min-Hyeon Han
}

Nexen Tire Corporation R\&D Center, Kyungnam 626-230, Republic of Korea

*Corresponding author: Fax: +82 55 3705366; Tel: +82 55 3705344; E-mail: ksw@ @exentire.co.kr

Rubber composites containing carbon black as a general fillers and multiwalled carbon nanotube, graphene and graphite as specific fillers were fabricated. Carbon materials were modified chemically and physically through an acid, heat and latex treatment to enhance the dispersion in rubber matrices. The acid, heat and latex treatment of carbon nanomaterials improved the mechanical properties, such as hardness, modulus, fatigue properties, electrical and thermal conductivity compared to pristine carbon materials. The modified graphene showed the most enhanced hardness, modulus and electrical conductivity but a significant decrease in the fatigue property and elongation. The modified carbon nanotube showed better thermal conductivity than the others, whereas the electrical and thermal conductivity of the nanocomposites decreased due to the formation of surface defects of multiwalled carbon nanotube by chopping.

Key Words: Carbon nanotube, Graphene, Graphite.

\section{INTRODUCTION}

Since carbon nanotube was discovered in 1991, carbon nanotubes and related carbon nanomaterials have attracted considerable attention because of their outstanding mechanical, electrical and thermal properties.

Carbon materials exist in many allotropes e.g., three dimensional crystals (diamond, graphite), two-dimensional graphene, one-dimensional nanotubes and zero-dimensional fullerenes ${ }^{1}$. Graphite is a layered crystal with c-axis lattice and carbon atoms within a layer bonded covalently. Graphite is well known for its high strength and high thermal conductivity and it offers the potential of fabricating multifunctional composites in a cost effective manner. carbon nanotubes are molecule-scale tubes of graphitic carbon with outstanding properties and exceptional mechanical, thermal and electrical properties. Graphene is defined as a flat monolayer of carbon atoms packed tightly into a two-dimensional honeycomb lattice. Along with its unique structure, graphene possesses a wide range of unusual properties. Most notably the thermal conductivity and mechanical stiffness are as high as the original values of graphite and the strength might be comparable to that of carbon nanotubes. Individual graphene has excellent electronic properties.

Nanocomposites of polymers containing previously mentioned carbon nanomaterials exhibited significant improvement in the properties of polymers and even generate novel properties that cannot be derived from their counterparts because the mixing of phases in them occurs over a much smaller length scale conpared to the micrometer length scale of conventional composites $^{2}$. Therefore, in recent years, nanoparticle based composites have attracted considerable attentions. Among them the most promising materials are the nanocomposites of carbon nanotube $^{3}$, graphene ${ }^{4}$ and exfoliated graphite . $^{5}$

Although the properties of carbon nanomaterials are excellent, they have not brought any remarkable improvements in the properties of composites because of their poor dispersion due to the large surface area and weak interaction with polymer chains, which causes large aggregations and clumps and as a result, significant decreases in hysteresis and elongation and fatigue properties ${ }^{6}$.

To obtain the expected properties, the improved dispersion of carbon nanomaterials within polymer matrices via increased interactions between the nanomaterials and polymer chains should be achieved. Many technical alternatives are available to enhance the dispersion of carbon nanomaterials.

First, there are physical treatments, such as ultrasonication, ball milling and heat treatment. Ultrasonication applies ultrasound energy to the mixture of solvent and nanomaterials over a period of several hours. Ball milling is a treatment to crush nanomaterials with a rotating ball. Heat treatment applies a 
high temperature thermal shock in nitrogen gas to nanomaterials. The advantages of these physical methods are the efficient elimination of impurities adsorbed on nanomaterials and the relatively easy achievement of a narrow distribution of length and diameter of nanomaterials and efficient exfoliation of graphite. On the other hand, such treatments enable nanomaterials to be dispersed only at the level of the aggregate units and result in a decrease in length and diameter as well as defects on the surface of the nanomaterials.

Chemical treatments, such as acid treatment, in-situ polymerization and latex treatment, are also used widely. An acid treatment involves the treatment of nanomaterials with nitric acid or sulphuric acid to functionalize them like $-\mathrm{C}=\mathrm{O}$, $-\mathrm{COOH}$ and $-\mathrm{OH}$ via in-situ polymerization, in which nanomaterials are added to the monomer solution followed by polymerization. A latex treatment was performed to mix the nanomaterials with polymer latex as dispersion media. The advantages of an acid treatment are the good dispersion of aggregates and functional groups to make nanomaterials interact with polymer chains, but this treatment results in more defects on the surface of the nanostructure than sonication. Ball milling has a huge negative effect on the electrical conductivity. In-situ polymerization and latex treatment can achieve better dispersion compared to other methods and has no defects on the surface of nanomaterials. The above chemical and physical treatment lead to a better dispersion and interaction with polymer chains but it is extremely difficult to produce treated carbon nanomaterials on a mass production scale. Therefore, the broader use of carbon nanotube and graphene is still limited due to the high cost.

This study was carried out to enhance the dispersion of carbon nanomaterials in rubber to improve various properties of rubber composites.

Rubber composites with nanofillers, such as multiwalled carbon nanotube, graphene and graphite, were fabricated and their mechanical, fatigue, electrical and thermal properties were examined. Carbon materials were modified chemically and physically to enhance their dispersion in rubber matrices.

\section{EXPERIMENTAL}

The matrix materials were a styrene butadiene rubber (SBR) with $23.5 \%$ bound styrene. A styrene butadiene latex ( $30 \%$ aqueous suspension and $70 \%$ SBR) was used as a dispersion media of nanofillers. The carbon-based nanofillers used in this study were carbon black N330 (OCI Co. Ltd.), entangled multiwalled carbon nanotube (E-MWNT, Carbon Nanotech Co. Ltd.), graphene (N-Baro Tech Co. Ltd.) and graphite (Timcal Co. Ltd.). N-tert-butyl- benzothiazole sulfonamide (TBBS), zinc oxide ( $\mathrm{ZnO})$, stearic acid (S/A), sulfur and processing oil were supplied by standard local manufacturers.

\section{Modification and treatment of carbon nanomaterials}

Latex treatment of multiwalled carbon nanotube, graphene and graphite: The carbon nanomaterials were added to the styrene butadiene rubber latex and stirred vigorously for $24 \mathrm{~h}$ at $80^{\circ} \mathrm{C}$ and the mixture was then coagulated with acetic acid, washed with water and dried at $100^{\circ} \mathrm{C}$.

Acid Treatment of multiwalled carbon nanotube and graphite: Nitric acid and sulphuric acid at the volume ratio (v/v) of 1:4 were mixed with graphite or multiwalled carbon nanotube at room temperature and stirred for $18 \mathrm{~h}$ and then washed with water until the solution became neutral. The samples were dried at $100{ }^{\circ} \mathrm{C}$ to remove the remaining moisture. This enables the surface of the multiwalled carbon nanotube and graphite to adopt functional groups, such as $-\mathrm{C}=\mathrm{O},-\mathrm{COOH}$ and $-\mathrm{OH}^{7}$.

Heat treatment of acid treated graphite: The acid treated graphite flakes were heated to $850{ }^{\circ} \mathrm{C}$ for $3 \mathrm{~min}$ in nitrogen gas to obtain an expanded or exfoliated structure.

\section{Characterization and measurement of properties}

Scanning electron microscopy (SEM, JEOL JSM-6490LV) was used to observe the morphology of the pristine carbon nanomaterials and fracture surface of composites.

Raman spectroscopy was used mainly to verify the nature of the carbon nanomaterials and the overall homogeneity of the sample. The Raman spectra, obtained in the range 2000$1000 \mathrm{~cm}^{-1}$, showed a band at $1588 \mathrm{~cm}^{-1}$ (G-band) due to the vibration mode and a band at $1357 \mathrm{~cm}^{-1}$ (D-band) arising from the disordered mode.

The curing characteristics were measured over $0.5 \mathrm{~h}$ period at $160{ }^{\circ} \mathrm{C}$ using a Oscillating disk rheometer (ODR) and the tensile properties were measured using a Universal Testing Machine (UTM, Instron Co., UK) at a crosshead speed of $300 \mathrm{~mm} / \mathrm{min}$.

The hardness was measured using shore A durometer (TECLOCK) and the fatigue properties of the composites were determined using a Demattia flex cracking tester (DMFC, UESHIMA).

The thermal conductivity was characterized by placing the probe on the sample surface using a thermal conductivity analyzer (QTM-500).

The electrical resistance of the rubber composites was examined at room temperature on a high voltage insulation resistance tester (TERAOHM, MI2077). At the interface between the probe tip and nanocomposites, two probes measured the electrical resistance.

\section{RESULTS AND DISCUSSION}

\section{Latex treatment}

Characterization of pristine materials: Fig. 1 shows SEM images of the carbon materials. Fig. 1a shows highly entangled and randomly organized E-MWNTs and Fig. 1b presents the worm-like shape of graphene, Fig. 1c presents the irregular honeycomb network of graphite sheets revealing the dense structures composed of many graphite sub-layers.

Fig. 2 and Table- 1 show the Raman spectra of carbon nanofillers. The so-called $\mathrm{G}$ and D bands, which appear at approximately 1588 and $1357 \mathrm{~cm}^{-1}$, respectively are the main features in the Raman spectra of the carbon nanofillers. All carbon nanofillers exhibited $G$ and $D$ bands and the $I(D) / I(G)$ ratio of graphene was higher than any other materials, suggesting minimal damage to graphite, which is in contrast to the significant devastation of graphene.

Characterization of the rubber nanocomposites mixed with carbon nanomaterials: Table- 2 lists the formulations of the styrene butadiene rubber composites with and without a latex treatment of carbon materials. All the carbon 

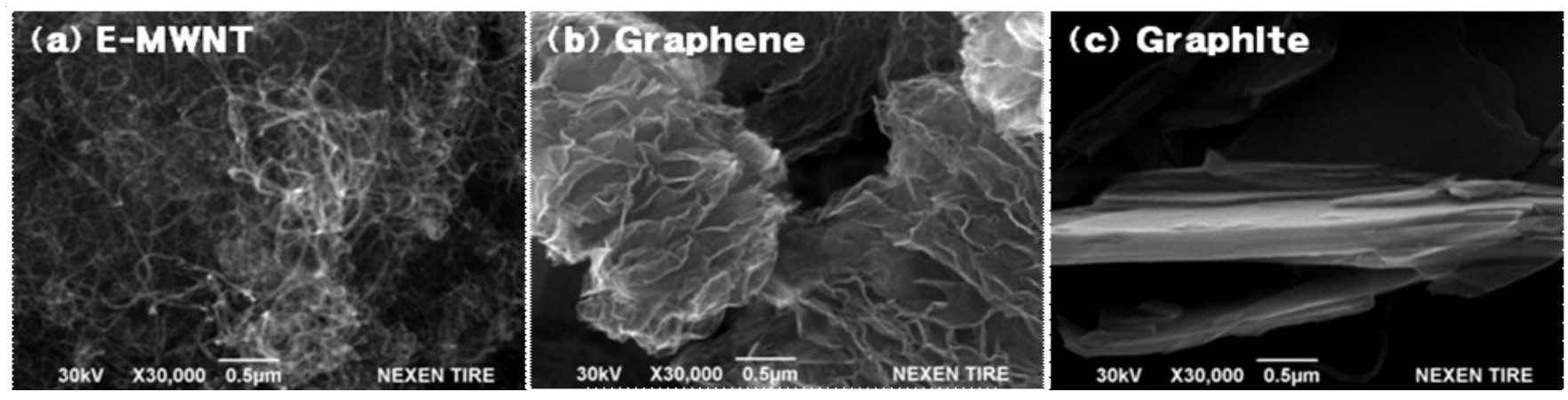

Fig. 1. SEM images of (a) E-MWNT, (b) Graphene and (c) Graphite
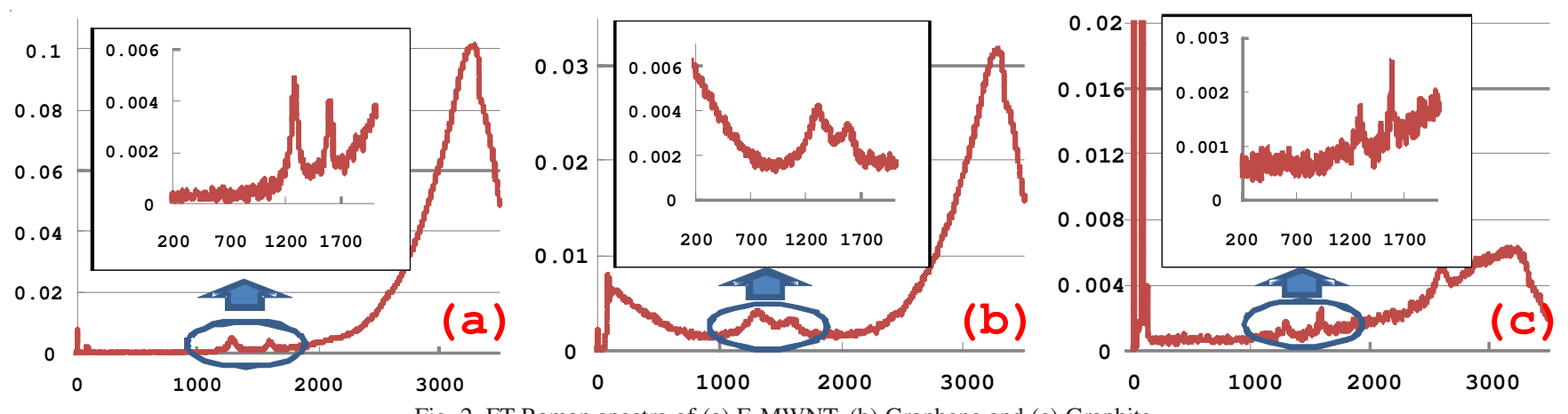

nanomaterial contents in the rubber composites were maintained at $3 \mathrm{phr}$.

\begin{tabular}{cccc}
\multicolumn{4}{c}{ TABLE-1 } \\
& $\begin{array}{c}\text { D BAND AND G BAND OF THE RAMAN } \\
\text { SPECTRA OF CARBON MATERIALS }\end{array}$ \\
\hline Material & D band & G band & $\mathrm{I}(\mathrm{D}) / \mathrm{I}(\mathrm{G})(\%)$ \\
\hline E-MWNT & 0.00492 & 0.00404 & 122 \\
Graphene & 0.00422 & 0.00337 & 125 \\
Graphite & 0.00337 & 0.0035 & 96 \\
\hline
\end{tabular}

\begin{tabular}{|c|c|c|c|c|c|c|c|c|c|c|}
\hline \multicolumn{11}{|c|}{$\begin{array}{c}\text { TABLE-2 } \\
\text { FORMULATIONS OF THE SBR COMPOSITES CONTAINING } \\
\text { CARBON MATERIALS WITH AND WITHOUT } \\
\text { LATEX TREATMENT }\end{array}$} \\
\hline \multirow{2}{*}{ Material } & \multicolumn{10}{|c|}{ Compound name (unit : phr) } \\
\hline & L1 & $\mathrm{L} 2$ & L3 & L4 & L5 & L6 & L7 & L8 & L9 & L10 \\
\hline E-SBR & 100 & 100 & 100 & 100 & 100 & 70 & 70 & 70 & 70 & 70 \\
\hline $\mathrm{C} / \mathrm{B}$ & 50 & 53 & 50 & 50 & 50 & 50 & 50 & 50 & 50 & 50 \\
\hline E-MWNT & - & - & 3 & - & - & - & - & - & - & - \\
\hline Graphene & - & - & - & 3 & - & - & - & - & - & - \\
\hline Graphite & - & - & - & - & 3 & - & - & - & - & - \\
\hline C/B-Latex & - & - & - & - & - & 33 & - & - & - & - \\
\hline MWNT-Latex & - & - & - & - & - & - & 33 & - & - & - \\
\hline Graphene-Latex & - & - & - & - & - & - & - & 33 & - & - \\
\hline Graphite-Latex & - & - & - & - & - & - & - & - & 33 & - \\
\hline SBR Latex & - & - & - & - & - & - & - & - & - & 30 \\
\hline
\end{tabular}

Table- 3 presents the curing properties of the carbon nanofillers/styrene butadiene rubber composites. The curing time of the rubber composites mixed with graphene was faster than the other rubber composites, regardless of the latex treatment. This was attributed to the more spacious specific surface by the two dimensions of graphene. The curing time of the rubber composites after the latex treatment became faster than without treatment because the presence of ammonium groups of styrene butadiene rubber latex facilitates the curing reactions. MH-ML, where ML is the lowest torque and $\mathrm{MH}$ is the highest torque at the curing curves, represents the crosslink density of the vulcanizates. MH-ML increases slightly after loading graphene indicating that the addition of graphene has a little effect on the crosslink density of rubber vulcanization. Table-3 lists the physical properties of the rubber composites. The composites with graphene showed a much higher reinforcing effect than the other fillers. The increased hardness and modulus were attributed to the highest specific surface area, whereas it showed a strong decrease in the elongation at break. The physical properties of the latex treated nanocomposites were better than those without treatment, which indicates that the dispersion of nanomaterials is increased by the latex dispersion media.

Fig. 3 shows the fracture surfaces of the rubber composites. The fracture surfaces of the rubber composites with graphene were rougher than the other surfaces of the composites corresponding to the physical properties.

Table-3 shows the swelling ratio and apparent crosslink density of the carbon nanocomposites with styrene butadiene rubber Latex. In the field of high polymers, there are many examples of materials that absorb large quantities of suitable solvents without dissolving. Vulcanized rubber, vinyl-divinyl copolymers and lightly gelled thermosetting resins, when placed in solvents, swell through imbibition to a degree depending on the solvent and polymer structure. The original shape was preserved and the swollen gel exhibited elastic behaviour rather than plastic properties. This phenomenon of limited swelling is characteristic of polymers possessing network structures. A state of equilibrium swelling may be attained if the network structure is permanent in the sense that its essential skeletal structure is not disrupted by the action of 


\begin{tabular}{|c|c|c|c|c|c|c|c|c|c|c|}
\hline \multicolumn{11}{|c|}{$\begin{array}{c}\text { TABLE-3 } \\
\text { PHYSICAL, THERMAL AND ELECTRICAL PROPERTIES OF THE NANOCOMPOSITES } \\
\text { WITH LATEX TREATED CARBON NANOMATERIALS }\end{array}$} \\
\hline & L1 & $\mathrm{L} 2$ & L3 & L4 & L5 & L6 & L7 & L8 & L9 & L10 \\
\hline $\mathrm{T} 40$ & 8.4 & 11.3 & 11.3 & 8.2 & 10.1 & 4.9 & 4.6 & 3.8 & 5.2 & 4.8 \\
\hline T90 & 16.6 & 20.6 & 20.7 & 16.8 & 18.9 & 10.8 & 11.9 & 11.2 & 11.1 & 11.0 \\
\hline Max Torque (Mpa) & 0.25 & 0.25 & 0.24 & 0.33 & 0.27 & 0.42 & 0.43 & 0.54 & 0.42 & 0.36 \\
\hline Min Torque (Mpa) & 0.08 & 0.08 & 0.07 & 0.1 & 0.10 & 0.19 & 0.22 & 0.31 & 0.19 & 0.17 \\
\hline$\Delta$ Torque $(\mathrm{Mpa})$ & 0.17 & 0.17 & 0.17 & 0.23 & 0.17 & 0.23 & 0.21 & 0.23 & 0.23 & 0.19 \\
\hline Hardness & 51 & 54 & 54 & 65 & 57 & 75 & 77 & 83 & 75 & 71 \\
\hline 200 \% Modulus (Mpa) & 4.5 & 4.9 & 4.5 & 8.3 & 5.1 & 14.9 & 13.8 & 23.1 & 14.0 & 10.9 \\
\hline Tensile strength (Mpa) & 18.5 & 17.5 & 17.3 & 17.4 & 16.8 & 27.7 & 26.3 & 24.5 & 28.4 & 27.8 \\
\hline Elongation $(\%)$ & 550 & 510 & 560 & 420 & 520 & 320 & 330 & 240 & 350 & 370 \\
\hline Q (Swelling ratio) & 4.78 & - & - & - & - & 4.61 & 4.51 & 4.19 & 4.42 & 4.66 \\
\hline 1/Q (crosslink density) & 0.209 & - & - & - & - & 0.216 & 0.221 & 0.239 & 0.226 & 0.215 \\
\hline Crack growth 2,000 cycles $(\mathrm{cm})$ & 2.19 & 2.24 & 2.27 & 3.21 & 2.28 & 3.45 & 3.28 & 4.03 & 3.26 & 3.02 \\
\hline Crack growth 6,000 cycles $(\mathrm{cm})$ & 2.86 & 2.76 & 3.03 & 5.97 & 2.98 & 3.94 & 3.66 & 5.36 & 3.47 & 3.82 \\
\hline Crack growth 10,000 cycles $(\mathrm{cm})$ & 3.67 & 3.81 & 3.95 & 8.17 & 4.35 & 4.43 & 4.91 & 8.43 & 5.66 & 4.88 \\
\hline Thermal conductivity (W/mK) & 0.276 & 0.282 & 0.293 & 0.286 & 0.289 & 0.318 & 0.353 & 0.332 & 0.337 & 0.307 \\
\hline Electrical resistivity $(\mathrm{K} \Omega)$ & 1070 & 425 & 249 & 31 & 146 & 265 & 43 & 9 & 119 & 914 \\
\hline
\end{tabular}
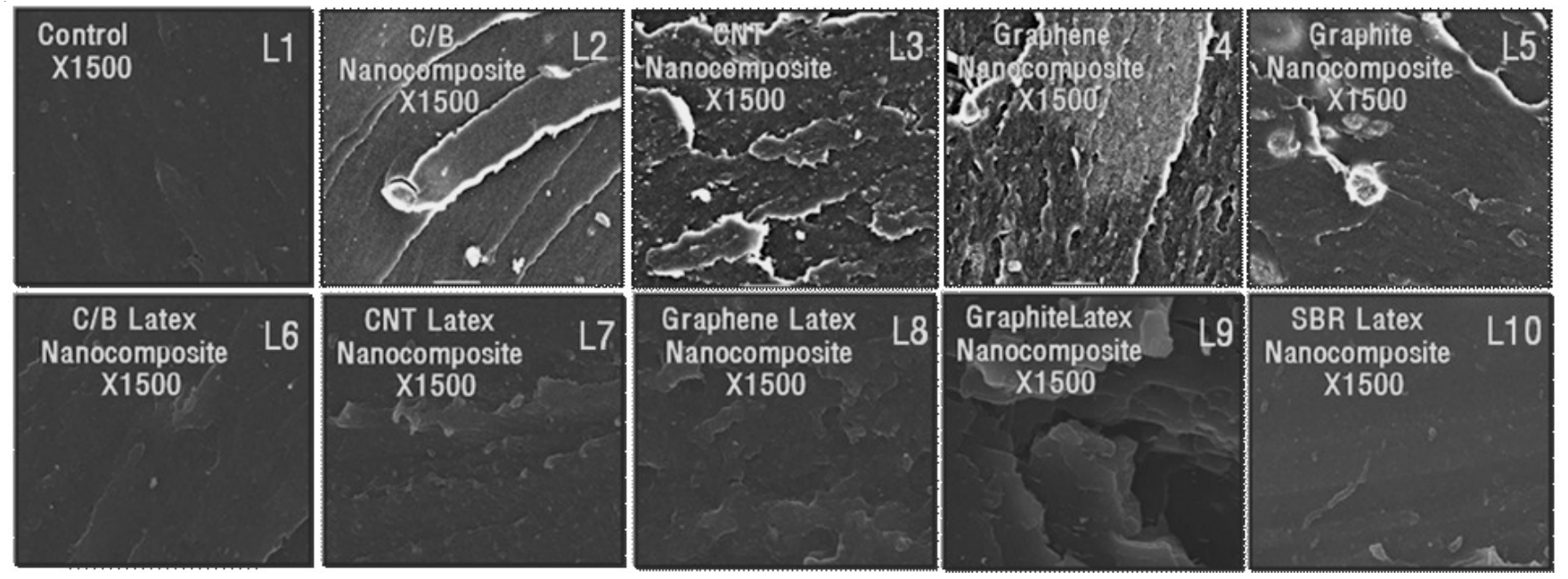

Fig. 3. SEM images of the fracture surface of nanocomposites with and without latex treated carbon nanomaterials

the solvent. As an increasing amount of solvent is absorbed by the polymer, the network is expanded progressively. The chains connecting the multifunctional network junction points are forced to assume more elongated, less probable, configurations. The swelling ratio Q of Eqn. (1) was recognized by the Flory-Rehner equation ${ }^{8}$. The calculated $\mathrm{Q}$ is representative of the degree of crosslinking of the rubber composites. This means that the reciprocal swelling ratio $\mathrm{Q}$ is generally proportional to the crosslinking rubber composites.

$$
\mathrm{Q}=(\mathrm{Wr} / \rho \mathrm{r}+\mathrm{Ws} / \mathrm{\rho s}) /(\mathrm{Wr} / \rho \mathrm{r})
$$

where $\mathrm{Q}$ is the ratio of swelling, $\mathrm{Wr}$ the weight of rubber composite, Ws is the weight of the absorbed solvent, $\rho$ r is the density of the rubber composite and $\rho$ s is the density of solvent.

The swelling ratio of the latex treated graphene nanocomposites is lower than any other latex treated nanocomposites, which shows good agreement with the fact that the hardness and modulus of the graphene latex nanocomposite are higher than the other nanocomposites.

Table- 3 shows the fatigue properties of the nanocomposites. Fatigue crack growth of the composites containing carbon nanofillers was similar except for the graphene composites. Fatigue crack growth of graphene is remarkably higher because the composites are more brittle than the others due to the higher hardness and modulus.

Table-3 also lists the thermal and electrical properties of the nanocomposites. Generally, the specific heat and thermal conductivity of carbon systems are determined primarily by phonons. At low temperatures, the phonon contribution to these quantities dominates and is due primarily to acoustic phonons. In addition, the phonon specific heat of graphene has a quadratic temperature contribution due to the longitudinal acoustic and in-plane transverse acoustic phonons and a linear contribution due to the out-of-plane transverse acoustic phonons. In addition, graphite is dominated by the three acoustic phonons. In the case of E-MWNT, carbon nanotubes yield a linear wave vector dependence of all four acoustic branches: longitudinal, twist and doubly-degenerate transverse ${ }^{9}$. A large aspect ratio of the materials is advantageous for the thermal conductivity within the composite as reported by Yu et al. ${ }^{10}$.

The electrical conducting mechanism of the polymer composites is associated with two questions: 1) how to form conducting paths and 2) how to conduct after the formation of these paths. Regarding the second question, several theories exist, such as contact conducting, tunneling effect, dielectric 
breakdown and field emission. The conductibility depends on their combined effect and can be summarized by the general statement that the microscopic migration of electrons might have several mechanisms. For example, percolation theory ${ }^{11}$, effective medium theory ${ }^{12}$, microstructure theory ${ }^{13}$ and thermodynamic theory ${ }^{14}$ are used to address the first question. These theories are related to the dispersed morphology and internal microstructure of the conducting fillers in the polymer matrices.

In addition to improving the mechanical properties, carbon nanofillers impart conductivity to elastomeric matrices. The electrical and thermal properties are improved by the addition of carbon nanofillers, which facilitate the electrical and thermal transition through filler-filler and filler-matrix interactions. In particular, the E-MWNT nanocomposites have the most predominant thermal conductivity regardless of adding the latex because E-MWNT transfers heat via four acoustic phonons and the higher aspect ratio of E-MWNT increases the probability of contact with each other. The composites with graphite have the next best thermal properties by three acoustic phonons and the composites using the graphene have the least improved thermal properties by the two acoustic phonons.

On the other hand, the electrical properties of the graphene composites were superior to the other composites, which suggest that graphene particles with large specific surfaces can abut or contact and form a conducting network, even at lower graphene contents. In addition, while E-MWNT and graphite have metallic and semi-conducting properties, graphene has metallic properties with a zero band gaps and can easily forms a conducting path.

Latex media enables rubber composites to obtain highly improved thermal and electrical properties without negative effects, indicating the increased dispersion of carbon nanofillers by latex.

\section{Acid and heat treatment}

Characterization of treated nanomaterials: Table-4 shows the Raman spectra of graphite and E-MWNT with and without treatment. The $\mathrm{I}(\mathrm{D}) / \mathrm{I}(\mathrm{G})$ ratio of the modified nanomaterials was higher than that of the untreated nanomaterials, which indicates that the amount of disorder or defects in the nanostructure are increased by the treatment. Such disorder and defects might involve functional groups on the graphite and E-MWNT surfaces, exfoliated graphite layers and destruction of the graphite and E-MWNT structure.
Characterization of carbon nanomaterial/rubber nanocomposite: Table-5 lists the formulations of the styrene butadiene rubber composites containing carbon materials with and without acid/heat treatment. All the carbon nanomaterial contents in the rubber composites were fixed to $5 \mathrm{phr}$.

Table- 6 shows the physical, thermal and electrical properties of the rubber composites mentioned above. The hardness and modulus of the nanocomposites with E-MWNT were higher than the composites with graphite due to the larger surface area and higher aspect ratio of the E-MWNT than the untreated multiwalled carbon nanotubes. Moreover, the acidtreated multiwalled carbon nanotubes showed lower tensile strength and elongation at break due to the poor interaction between E-MWNT and rubber chains indicating that the acid treatment of multiwalled carbon nanotube is barely suitable for improving the mechanical properties. An acid or acid/heat treatment of graphite increases the modulus and tensile strength compared to untreated graphite, showing that the treatment of graphite causes a higher surface area and lower thickness of graphite layers.

Fatigue crack growth of modified graphite is lower than other composites. This shows that the tearing energy is decreased

\begin{tabular}{|c|c|c|c|c|}
\hline \multicolumn{5}{|c|}{$\begin{array}{c}\text { TABLE-4 } \\
\text { D AND G BAND OF RAMAN SPECTRA OF THE CARBON } \\
\text { MATERIALS WITH AND WITHOUT TREATMENT }\end{array}$} \\
\hline Material & Treatment & D band & G band & $\mathrm{I}(\mathrm{D}) / \mathrm{I}(\mathrm{G})(\%)$ \\
\hline \multirow{3}{*}{ Graphite } & - & 0.00337 & 0.0035 & 96 \\
\hline & Acid & 0.00206 & 0.00099 & 208 \\
\hline & Acid \& Heat & 0.0024 & 0.00091 & 264 \\
\hline \multirow{2}{*}{ E-MWNT } & - & 0.00492 & 0.00404 & 122 \\
\hline & Acid & 0.00388 & 0.00269 & 144 \\
\hline
\end{tabular}

\section{TABLE-5}

FORMULATIONS OF THE SBR COMPOSITES CONTAINING CARBON MATERIALS WITH AND WITHOUT ACID AND HEAT TREATMENT

\begin{tabular}{lccccccc}
\hline & \multicolumn{7}{c}{ Compound Name (unit : phr) } \\
\hline Material & A1 & A2 & A3 & A4 & A5 & A6 & A7 \\
\hline E-SBR & 100 & 100 & 100 & 100 & 100 & 70 & 70 \\
C/B & 50 & 55 & 50 & 50 & 50 & 50 & 50 \\
Graphite & - & - & 5 & - & - & - & - \\
Acid-graphite & - & - & - & 5 & - & - & - \\
Acid \& heat-graphite & - & - & - & - & 5 & - & - \\
E-MWNT & - & - & - & - & - & 5 & - \\
Acid-MWNT & - & - & - & - & - & - & 5 \\
\hline
\end{tabular}

Carbon nanomaterials contents in rubber compound: $5 \mathrm{phr}$

TABLE-6

PHYSICAL, THERMAL AND ELECTRICAL PROPERTIES OF THE NANOCOMPOSITES CONTAINING CARBON NANOMATERIALS WITH AND WITHOUT ACID/HEAT TREATMENT

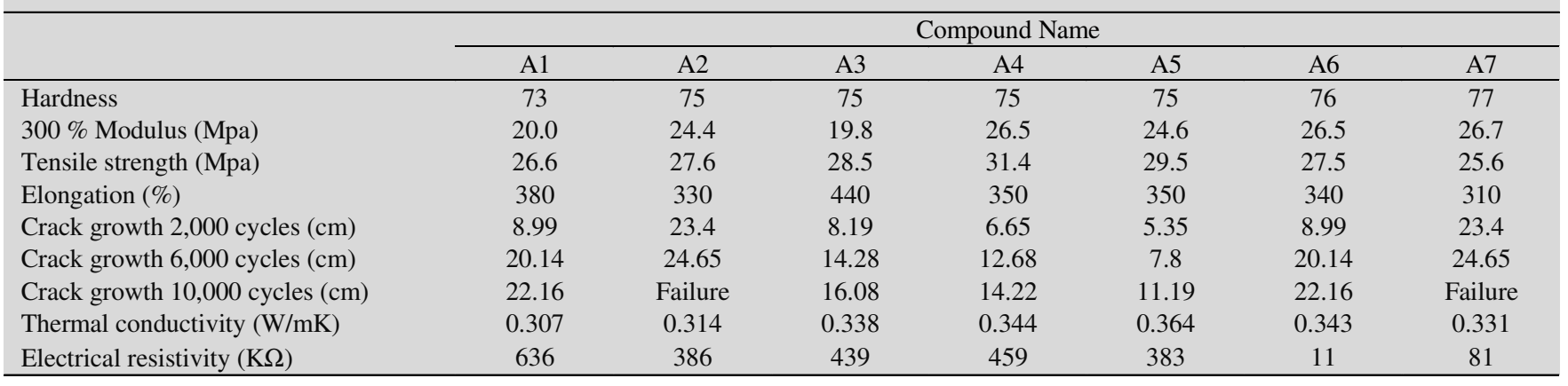


and fatigue life is increased due to improved dispersion and interaction between functionalized and expanded graphite and rubber chains.

The acid and heat treatment of graphite improved the thermal and electrical properties of the nanocomposites, showing the highest thermal conductivity. This was attributed to the improved dispersion and interaction between expanded graphite and rubber chains. In the case of the electrical resistivity, the unmodified E-MWNT showed the best electric conductivity, whereas the acid-treated E-MWNT showed lower thermal and electrical properties. This suggests that the acid treatment of E-MWNT causes a decrease in length and defects on the surface and/or nanostructure, which has an adverse effect on the thermal and electrical properties.

\section{Conclusion}

Carbon nanofillers/styrene butadiene rubber latex composites were prepared and their mechanical, fatigue, thermal and electrical properties were evaluated according to the fillers. The latex nanocomposites showed substantially improved mechanical properties due to the increased polymer-filler interactions and filler dispersions and enhanced electrical and thermal properties due to the increased formation of connected filler structures. In particular, composites containing graphene exhibited a higher modulus, hardness and better electrical properties than the other nanocomposites. In the swelling result, the latex-treated nanocomposites using graphene showed less swelling than the other latex treated nanocomposites, which is consistent with the higher hardness and modulus of graphene latex nanocomposite. In addition, the fracture surface of the nanocomposites with graphene was rougher than those with carbon black or graphite. The composite containing E-MWNT had the best thermal properties due to the four acoustic phonons, while the electrical properties of composites containing graphene were better than the other composites due to metallic properties by zero band gaps. Thermal and electrical properties of latex-treated nanocomposites were better than the nanocomposites without treatment, which was attributed to the increased dispersion of carbon nanofiller by the latex dispersion media.

The effects of the acid and heat treatments on the nanocomposite properties were also investigated. Acid and heat treatment generates functional groups and expanded the structure of graphite, which facilitated both physical and chemical interactions between graphite surface and polymer chain. Consequentially, the treatments improved physical, thermal, electrical and fatigue properties without adverse effects except for elongation. In contrast, acid treatment of multiwalled carbon nanotube generates defects on the multiwalled carbon nanotube structure and a shorter length. Therefore it has few positive effects on the mechanical properties and a significantly negative effect on the electrical properties.

\section{REFERENCES}

1. M.I. Katsnelson, Mater. Today, 10, 20 (2007).

2. M. Sumita, T. Tsukumo, K. Miyasaka and K. Ishikawa, J. Mater Sci., 18, 1758 (1983).

3. S. Wang, R. Liang, B. Wang and C. Zhang, Carbon, 47, 53 (2009).

4. A. Yasmin and I.M. Daniel, Polymer, 45, 8211 (2004).

5. H. Kim and C.W. Macosko, Polymer, 50, 3797 (2009).

6. F. Cataldo, O. Ursini and G. Angelini, Fullerenes, Nanotubes and Carbon Nanostructures, 17, 38 (2009).

7. G. Chen, C. Wu, W. Weng, D. Wu and W. Yan, Polymer, 44, 1781 (2003).

8. P.J. Flory and J. Rehner, J. Chem. Phys., 11, 512 (1943).

9. V.N. Popov, J. Mater. Sci., 43, 61 (2004).

10. A. Yu, P. Ramesh, M.E. Itkis, E. Bekyarova and R.C. Haddon, J. Phys. Chem. C, 111, 7565 (2007).

11. C. Zhang, X. Yi, H. Yui, S. Asai and M. Sumita, J. Appl. Polym. Sci., 69, 1813 (1998).

12. D.S. Mclachaln, Solid State Phys., 21, 1521 (1988),

13. M. Weber and M.R. Kamal, Polym. Comp., 18, 711 (1997).

14. F. Lux, J. Mater. Sci., 28, 285 (1993). 